\title{
Oligometastatic head and neck cancer: Which patients benefit from radical local treatment of all tumour sites?
}

\author{
Thomas Weissmann', Daniel Höfler ${ }^{1}$, Markus Hecht ${ }^{1}$, Sabine Semrau ${ }^{1}$, Marlen Haderlein ${ }^{1}$, Irina Filimonova ${ }^{1}$, \\ Benjamin Frey ${ }^{1}$, Christoph Bert ${ }^{1}$, Sebastian Lettmaier ${ }^{1}$, Konstantinos Mantsopoulos ${ }^{2}$, Heinrich Iro², \\ Rainer Fietkau ${ }^{1}$ and Florian Putz ${ }^{1 *}$ (D)
}

\begin{abstract}
Background: There is a large lack of evidence for optimal treatment in oligometastatic head and neck cancer and it is especially unclear which patients benefit from radical local treatment of all tumour sites.

Methods: 40 patients with newly diagnosed oligometastatic head and neck cancer received radical local treatment of all tumour sites from 14.02.2008 to 24.08.2018. Primary endpoint was overall survival. Time to occurrence of new distant metastases and local control were evaluated as secondary endpoints as well as prognostic factors in univariate und multivariate Cox's regression analysis. To investigate the impact of total tumour volume on survival, all tumour sites were segmented on baseline imaging.

Results: Radical local treatment included radiotherapy in $90 \%$ of patients, surgery in $25 \%$ and radiofrequency ablation in 3\%. Median overall survival from first diagnosis of oligometastatic disease was 23.0 months, 2-year survival was $48 \%$, 3-year survival was 37\%, 4-year survival was $24 \%$ and 5 -year survival was $16 \%$. Median time to occurrence of new distant metastases was 11.6 months with freedom from new metastases showing a tail pattern after 3 years of followup (22\% at 3, 4- and 5-years post-treatment). In multivariate analysis, better ECOG status, absence of bone and brain metastases and lower total tumour volume were significantly associated with improved survival, whereas the number of metastases and involved organ sites was not.

Conclusions: Radical local treatment in oligometastatic head and neck cancer shows promising outcomes and needs to be further pursued. Patients with good performance status, absence of brain and bone metastases and low total tumour volume were identified as optimal candidates for radical local treatment in oligometastatic head and neck cancer and should be considered for selection in future prospective trials.
\end{abstract}

Keywords: Oligometastatic disease, Local treatment, Radiotherapy, SBRT, Head and neck cancer

*Correspondence: florian.putz@uk-erlangen.de

${ }^{1}$ Department of Radiotherapy, Friedrich-Alexander-Universität ErlangenNürnberg, Universitaetsstraße 27, 91054 Erlangen, Germany

Full list of author information is available at the end of the article

\section{Background}

Oligometastatic disease is an increasingly recognized disease entity in metastatic solid malignancies characterized by limited metastatic burden and consecutive benefit of local treatment to all metastatic sites [1-3]. Introduced at a conceptual level as early as 1995 by Hellman and Weichselbaum [4], it was in very recent years that prospective randomized Phase II trials increasingly provided original author(s) and the source, provide a link to the Creative Commons licence, and indicate if changes were made. The images or other third party material in this article are included in the article's Creative Commons licence, unless indicated otherwise in a credit line to the material. If material is not included in the article's Creative Commons licence and your intended use is not permitted by statutory regulation or exceeds the permitted use, you will need to obtain permission directly from the copyright holder. To view a copy of this licence, visit http://creativecommons.org/licenses/by/4.0/. The Creative Commons Public Domain Dedication waiver (http://creativeco mmons.org/publicdomain/zero/1.0/) applies to the data made available in this article, unless otherwise stated in a credit line to the data. 
empirical evidence for improved outcome with radical local treatment to all tumour sites in cohorts consisting mainly of oligometastatic non-small cell lung cancer (NSCLC) and prostate cancer patients [5-8]. While these Phase II trials by design were not able to provide confirmatory evidence, they strongly indicated a systemic effect of local treatment. Among other studies, the wellrecognized Phase II trial by Gomez et al., reported significantly improved overall survival (41.2 vs. 17 months) as well as decreased occurrence of new distant lesions in oligometastatic NSCLC patients [5]. Encouraged by such promising results, scientific societies like the EORTC, ESTRO and ASTRO have put forth diagnostic criteria as well as classification proposals for oligometastatic disease in recent months $[1,2]$. Despite these efforts, oligometastatic disease remains poorly defined and the most widely recognized criterion of oligometastatic disease is the ability to safely apply local treatment to all tumour sites, which is largely because of a great lack of scientific studies to base any additional criteria upon. As such it is currently still largely unclear, which patients should receive radical local treatment of all metastatic sites in addition to or instead of systemic treatment for metastatic disease. This is especially true in metastatic head and neck cancers, in which the significance and potential benefit of radical local treatment remains largely unexplored to this date and only very few and small series have been published so far. Although investigation of the oligometastatic paradigm is particularly challenging in metastatic head and neck cancers, any resulting contribution to current systemic treatment options for patients with metastatic disease could be of particular value.

Starting from 2008 and as a multi-disciplinary endeavour, patients with metastatic head and neck cancers received local treatment to all tumour sites as part of routine clinical care at the University Hospital Erlangen, as long as safe application of local treatment to all locations was ensured. In the present work we report the clinical results and treatment details of this cohort. Furthermore, we explore potential prognosticators including tumour volume-based metrics that have been implicated in the definition of oligometastatic disease to further elucidate which patients with metastatic head and neck cancer will benefit the most from radical local treatment of all tumour sites.

\section{Methods}

\section{Patient population}

Patients with metastatic head and neck cancer received ablative local treatment to all tumour sites at the University hospital Erlangen as part of routine clinical care. Patients were selected for radical local treatment, if all tumour sites could be safely treated locally, but no formal thresholds e.g., for number of metastases or involved organ sites was used. This unique setting enabled us to investigate prognostic factors that could improve patient selection and optimize the definition of oligometastatic disease in head and neck cancers. For this retrospective analysis we identified forty head and neck cancer patients who had been first diagnosed with synchronous or metachronous metastases and subsequently received radical local treatment of all tumour sites irrespective of the number of metastases or involved organ sites. According to the recent ESTRO/EORTC classification, $68 \%(27 / 40)$ of these patients suffered from metachronous oligorecurrence, whereas $33 \%(13 / 40)$ had synchronous oligometastatic disease [2]. 30\% (12/40) had an active locoregional tumour manifestation in addition to distant metastases. 55\% (22/40) of patients had histologic proof of metastatic disease, while $45 \%$ (18/40) had imaging diagnosis of metastases alone.

\section{Treatment}

Local treatments were recommended after joint interdisciplinary review by experts in radiation oncology, head and neck surgery, interventional radiology as well as thoracic and visceral surgery within the framework of an interdisciplinary tumour board with recommendations being based on patient- and disease-specific as well as technical considerations with the aim to achieve local ablation of each tumour site in the safest possible manner. In total $90 \%(36 / 40)$ of patients received radiotherapy as part of their treatment for oligometastatic disease (OMD), 25\% (10/40) of patients received surgery and 1 patient $(3 \%)$ received radiofrequency ablation of liver metastases. A detailed description of local treatments by individual patient case is provided in Additional file 1: Table S1. Patients routinely received restaging using computed tomography 6 weeks after local treatment and then subsequently at intervals of 3 months.

Regarding systemic treatment, 35\% (14/40) of patients received platinum-based combination treatment, $20 \%$ $(8 / 40)$ received single agent cytostatic chemotherapy or cetuximab alone, $5 \%(2 / 40)$ were treated with cetuximab + platinum combination therapy and 5\% (2/40) received immune checkpoint inhibitor treatment. Systemic treatment was deferred in 14 patients with a solitary metastasis, who received imaging follow-up at close intervals following local treatment of metastatic disease (Table 1).

\section{Volumetric analysis}

In baseline imaging all tumour sites were semiautomatically segmented in every patient using the NVIDIA Clara AI-assisted annotation extension for the OpenSource software 3D Slicer v.4.11.0 [9], which 
Table 1 Patient characteristics at first local treatment

\begin{tabular}{|c|c|}
\hline Parameter & Total cohort $(\mathrm{N}=40)$ \\
\hline \multicolumn{2}{|c|}{ ESTRO/EORTC Type of oligometastatic disease, n (\%) } \\
\hline Metachronous Oligorecurrence & $27(68 \%)$ \\
\hline Synchronous Oligometastatic disease & $13(33 \%)$ \\
\hline \multicolumn{2}{|l|}{ Age, years } \\
\hline Median (IQR) & $60.5(56.3-70.8)$ \\
\hline Mean (range) & $62.0(41.0-82.0)$ \\
\hline \multicolumn{2}{|l|}{ ECOG, n (\%) } \\
\hline ECOG 0 & $5(13 \%)$ \\
\hline ECOG 1 & $18(45 \%)$ \\
\hline ECOG 2 & $14(35 \%)$ \\
\hline ECOG 3 & $3(8 \%)$ \\
\hline \multicolumn{2}{|l|}{ Histology, n (\%) } \\
\hline Squamous cell carcinoma & $33(83 \%)$ \\
\hline Lymphoepithelial carcinoma & $3(8 \%)$ \\
\hline Adenocarcinoma & $2(5 \%)$ \\
\hline Neuroendocrine carcinoma & $1(3 \%)$ \\
\hline Undifferentiated & $1(3 \%)$ \\
\hline \multicolumn{2}{|l|}{ Original site of Head and Neck primary, n (\%) } \\
\hline Hypopharynx & $11(28 \%)$ \\
\hline Larynx & $9(23 \%)$ \\
\hline Oropharynx & $7(18 \%)$ \\
\hline Head and neck cancer of unknown primary & $5(13 \%)$ \\
\hline Nasal cavity/paranasal sinuses & $4(10 \%)$ \\
\hline Oral cavity & $3(8 \%)$ \\
\hline Nasopharynx & $1(3 \%)$ \\
\hline \multicolumn{2}{|l|}{ Number of metastases } \\
\hline Median (IQR) & $1.0(1.0-2.0)$ \\
\hline Mean (range) & $1.6(1.0-7.0)$ \\
\hline \multicolumn{2}{|l|}{ Metastatically involved organ systems, n (\%) } \\
\hline One organ system & $34(85 \%)$ \\
\hline Two organ systems & $6(15 \%)$ \\
\hline \multicolumn{2}{|l|}{ Metastatically involved organ sites ${ }^{\mathrm{a}}, \mathrm{n}(\%)$} \\
\hline Pulmonary & $23(58 \%)$ \\
\hline Lymphonodal & $11(28 \%)$ \\
\hline Bone & $6(15 \%)$ \\
\hline Hepatic & $3(8 \%)$ \\
\hline Brain & $2(5 \%)$ \\
\hline \multicolumn{2}{|l|}{ Total tumor volume, $\mathrm{cm}^{3}$} \\
\hline Median (IQR) & $19.7(2.0-46.8)$ \\
\hline Mean (range) & $49.5(0.2-550.8)$ \\
\hline \multicolumn{2}{|l|}{ Total metastases volume, $\mathrm{cm}^{3}$} \\
\hline Median (IQR) & $9.4(1.6-23.2)$ \\
\hline Mean (range) & $24.5(0.2-240.6)$ \\
\hline \multicolumn{2}{|c|}{ Number of active tumor sites (metastases + primary) } \\
\hline Median (IQR) & $2.0(1.0-2.0)$ \\
\hline Mean (range) & $1.8(1.0-7.0)$ \\
\hline \multicolumn{2}{|l|}{ Locoregional tumor, n (\%) } \\
\hline No & $28(70 \%)$ \\
\hline Primary manifestation & $7(18 \%)$ \\
\hline
\end{tabular}


Table 1 (continued)

\begin{tabular}{|c|c|}
\hline Parameter & Total cohort $(\mathrm{N}=40)$ \\
\hline Recurrence & $5(13 \%)$ \\
\hline \multicolumn{2}{|l|}{ Locoregional tumor site, $\mathrm{n}(\%)$} \\
\hline Primary and involved regional lymph nodes & $7(18 \%)$ \\
\hline Regional lymph nodes only & $3(8 \%)$ \\
\hline Primary only & $2(5 \%)$ \\
\hline \multicolumn{2}{|l|}{ Total locoregional tumor volume, $\mathrm{cm}^{3}$} \\
\hline Median (IQR) & $0.0(0.0-7.0)$ \\
\hline Mean (range) & $24.9(0.0-502.4)$ \\
\hline \multicolumn{2}{|l|}{ Previous head and neck radiotherapy, $\mathrm{n}(\%)$} \\
\hline Prior head and neck radiotherapy & $31(78 \%)$ \\
\hline No prior head and neck radiotherapy & $9(23 \%)$ \\
\hline \multicolumn{2}{|l|}{ Previous head and neck surgery, $\mathrm{n}(\%)$} \\
\hline Prior head and neck surgery & $23(58 \%)$ \\
\hline No prior head and neck surgery & $17(43 \%)$ \\
\hline \multicolumn{2}{|l|}{ Histologic proof of metastatic disease, $n(\%)$} \\
\hline Yes & $22(55 \%)$ \\
\hline No & $18(45 \%)$ \\
\hline \multicolumn{2}{|l|}{ FDG-PET staging, $n(\%)$} \\
\hline Yes & $13(33 \%)$ \\
\hline No & $27(68 \%)$ \\
\hline \multicolumn{2}{|c|}{ Interval from diagnosis of OMD to first local treatment, months } \\
\hline Median (IQR) & $1.3(0.9-2.2)$ \\
\hline Mean (range) & $1.7(0-7.3)$ \\
\hline \multicolumn{2}{|l|}{ Total duration of OMD first-line treatment, months } \\
\hline Median (IQR) & $3.1(2.2-4.6)$ \\
\hline Mean (range) & $3.7(0.3-10.8)$ \\
\hline \multicolumn{2}{|l|}{ Local treatment for OMDa, $\mathrm{n}(\%)$} \\
\hline Radiotherapy & $36(90 \%)$ \\
\hline Surgery & $10(25 \%)$ \\
\hline Interventional radiology & $1(3 \%)$ \\
\hline \multicolumn{2}{|l|}{ Biologically effective dose $(a / \beta=10)^{b}$, Gy } \\
\hline Median (IQR) & $78.8(67.2-111.4)$ \\
\hline Mean (range) & $82.6(39.0-115.2)$ \\
\hline \multicolumn{2}{|l|}{ Systemic treatment, $\mathrm{n}(\%)$} \\
\hline Platinum-combination treatment & $14(35 \%)$ \\
\hline Single-agent cytostatic chemotherapy alone & $4(10 \%)$ \\
\hline Cetuximab alone & $4(10 \%)$ \\
\hline Platinum-combination + Cetuximab & $2(5 \%)$ \\
\hline Immune checkpoint inhibitor & $2(5 \%)$ \\
\hline No concurrent systemic treatment & $14(35 \%)$ \\
\hline
\end{tabular}

$O M D$ oligometastatic disease

a Some patients are part of multiple categories

b Minimum biologically effective dose to tumor locations in patients that received radiotherapy

is a neural-network based autosegmentation solution. All autosegmentations were then manually validated and corrected by an experienced radiation oncologist.
Total mesh-based tumour volumes were calculated from these segmentations using SlicerRadiomics [10]. 
3D Renderings and slice-based representations were created using 3DSlicer v.4.11.0.

\section{Statistical analysis}

Primary endpoint was overall survival. Time to occurrence of new distant metastases and local control were evaluated as secondary endpoints as well as prognostic factors in univariate und multivariate Cox's regression analysis.

The number of metastases and involved organ sites was counted in baseline imaging with multiple lymph node metastases in one lymph node region (e.g., multiple mediastinal lymph nodes) being counted as one distant metastasis. Overall survival was calculated from the first diagnosis of oligometastatic disease, i.e., date of first imaging showing metastases, until death or censored at last follow-up. Time to new distant metastases was similarly determined from first diagnosis of oligometastatic disease to occurrence of new distant metastases or censored at death or last follow-up. Local control was calculated at a lesion-level from the date of first local treatment for oligometastatic disease until progression according to RECIST 1.1 criteria [11] or censored at last follow-up or death. Time to event outcomes were assessed using the Kaplan-Meier method and the logrank test. Prognostic factors were first assessed using univariate Cox's regression analysis and significant prognosticators $(p<0.05)$ from univariate analysis were then included in the multivariate Cox's regression model. Statistics were calculated using SPSS 21.0, Graphs were created using GraphPad Prism 8.4.

\section{Results}

A total of 40 patients with newly diagnosed oligometastatic head and neck cancer received ablative local treatment to all tumour sites. Median age was 60.5 years (range, 41-82 years). Median number of metastases was 1 (range, 1-7) with the lung being the most commonly affected organ site (58\%). 30\% of patients (12/40) had active locoregional tumour in addition to distant metastases. The median interval from first imaging diagnosis of oligometastatic disease (OMD) to start of first local treatment (i.e., first local treatment of metastases or locoregional tumour manifestations) was 1.3 months. The median duration to subsequently complete all local treatments was 3.1 months. 90\% (36/40) of patients received radiotherapy as part of their treatment for OMD, $25 \%$ $(10 / 40)$ of patients received surgery and 1 patient $(3 \%)$ received radiofrequency ablation of liver metastases. 73\% $(29 / 40)$ of patients were treated exclusively with radiotherapy for OMD. Full details concerning cohort characteristics and treatment schedules are listed in Table 1 and Additional file 1: Table S1. Total tumour volume was determined for all patients via segmentation of all tumour sites in imaging studies at the time of first diagnosis of OMD (Fig. 1).

After a median follow-up of 65.2 months 83\% (33/40) of patients had died. Median overall survival from first diagnosis of oligometastatic disease was 23.0 months. 1-year overall survival was $70 \%, 2$-year survival was $48 \%$, 3-year survival was $37 \%$, 4-year survival was $24 \%$ and 5 -year survival was $16 \%$ (Fig. 2a). In the subgroup of patients that were exclusively treated with radiotherapy
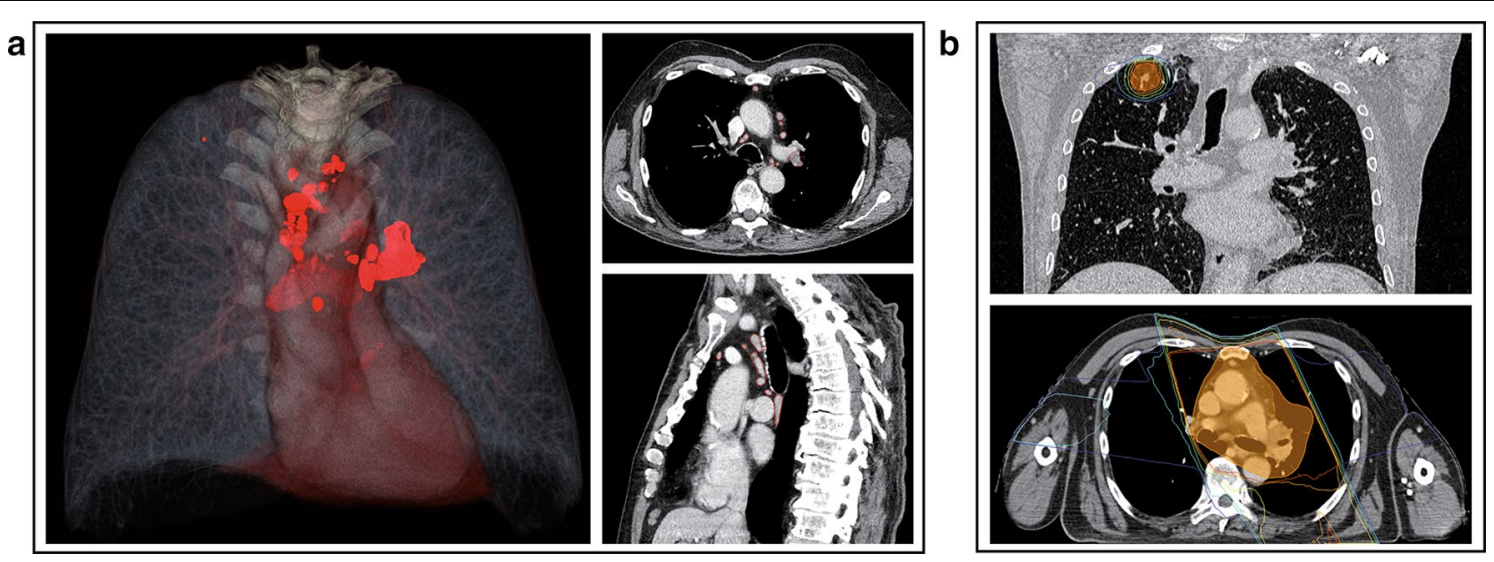

Fig. 1 Total tumour volume (a) and corresponding radiotherapy treatment plans (b) in a patient with mediastinal lymph node metastases and a single pulmonary lesion from metastatic laryngeal cancer. a: All tumour sites were analysed volumetrically via tumour segmentations in all patients to obtain total tumour volumes at diagnosis of oligometastatic disease. Left: 3D rendering showing segmented mediastinal lymph node metastases and a single right-upper lobe metastasis (red). Right: Axial and sagittal view of segmented lymph node metastases. b: Radiotherapy treatment plan showing isodoses (red: $95 \%$, orange: $90 \%$, yellow: $80 \%$, green: $60 \%$, cyan: $40 \%$ and blue: $30 \%$ ) and planning target volumes of stereotactic body radiotherapy to the right-upper lobe metastasis $(12 \times 6 \mathrm{~Gy})$ as well as of conventionally fractionated chemoradiation $(25 \times 1.8 \mathrm{~Gy}+12 \times 1.8 \mathrm{~Gy}$ Boost [not shown]) of mediastinal lymph node metastases 
a

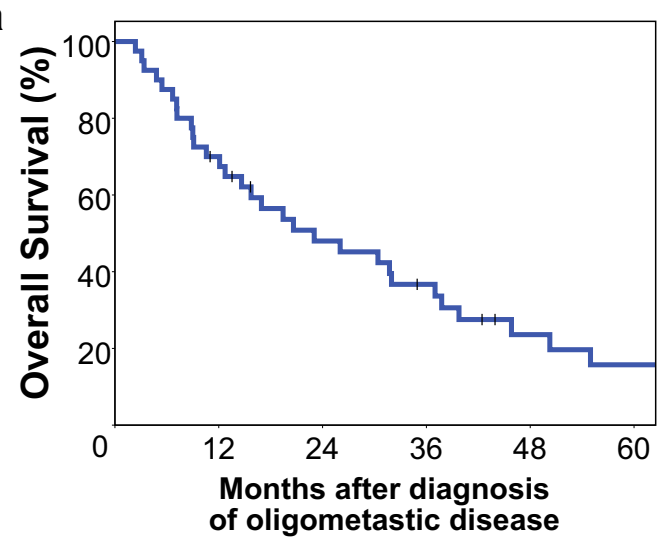

C

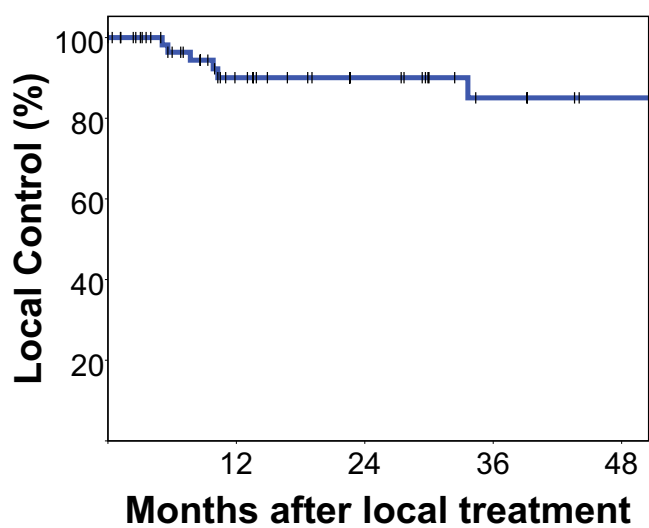

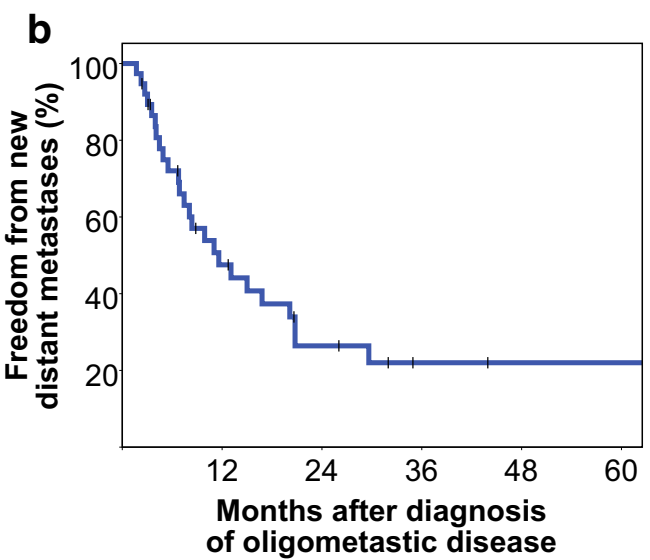

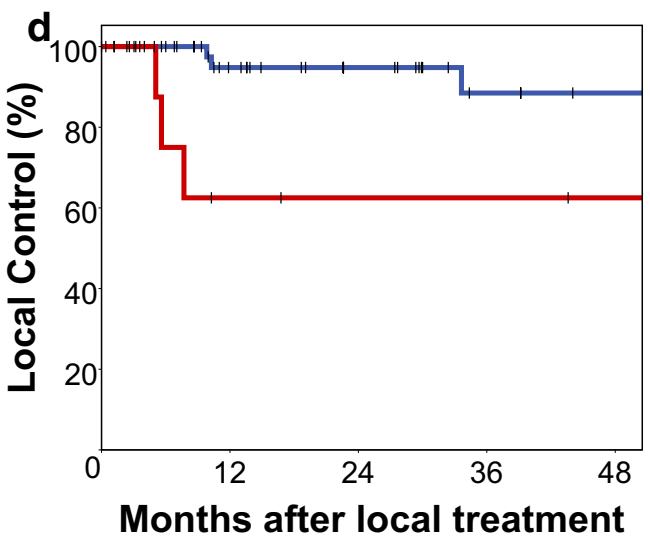

Fig. 2 a Kaplan-Meier plot showing overall survival since first diagnosis of oligometastatic disease and $\mathbf{b}$ freedom from new distant metastases in all 40 patients. $\mathbf{c}$ Local control in all of the 75 treated tumour sites and $\mathbf{d}$ local control for locoregional tumour manifestations versus distant metastases

( $\mathrm{n}=29$ ), median overall survival was 20.6 months. 1-year overall survival was $69 \%$, 2-year survival was $45 \%$, 3-year survival was $37 \%$, 4 -year survival was $23 \%$ and 5 -year survival was $15 \%$.

Median time to occurrence of new distant metastases was 11.6 months with 1-year freedom from distant metastases being 48\%, 2-year freedom from new distant metastases being $26 \%$ and 3-year freedom from new distant metastases being $22 \%$. Interestingly, freedom from new distant metastases subsequently ceased to decline and showed a tail pattern with $22 \%$ of patients remaining free from new distant metastases at 4- and 5-years post diagnosis of oligometastatic disease (Fig. 2b). Similar findings were obtained in the subgroup exclusively treated with radiotherapy. In patients treated exclusively with radiotherapy, median time to occurrence of new distant metastases was 9.9 months with 1 -year freedom from distant metastases being 45\%, 2-year freedom from new distant metastases being 35\% and 3-year, 4-year and 5 -year freedom from new distant metastases being $29 \%$.
Regarding local treatment effect, 8 out of a total of 75 lesions showed progression after a median imaging follow-up of 19.1 months. 1-year and 2-year local control was $90 \%$, 3-year and 4-year local control was $85 \%$. There was no difference between treatment modalities (logrank $p=0.324)$. Local control was higher for metastases than for locoregional head and neck tumour manifestations without reaching significance, however (1-year local control $95 \%$ vs. $63 \%, p=0.122$, Fig. 2 c, d). These locoregional tumour manifestations were primary tumours in $58 \%(7 / 12)$ and recurrent disease in $42 \%$ (5/12). Radical local treatment of locoregional tumour manifestations was surgery alone in $25 \%(3 / 12)$, chemoradiation in $58 \%$ $(7 / 12)$ and surgery followed by chemoradiation in $17 \%$ $(2 / 12)$. All treatments and tumour sites are reported in full detail in Additional file 1: Table S1 at an individual patient level.

Despite numerically worse local control for locoregional manifestations, overall survival was not significantly different for patients with and without active 
locoregional tumour manifestations $(p=0.574$, see Table 2 and below). In a subgroup of 35\% (14/40) patients with solitary metastasis, systemic treatment was deferred after local treatment. 79\% (11/14) of these patients had pulmonary metastasis. In this subgroup without initial systemic treatment, 1-year systemic treatment-free survival was $50 \%$ with 2 - and 3 -year systemic treatment-free survival being $36 \%$ and $14 \%$, respectively (Fig. 3 ).

To determine which patients with metastatic disease benefit from radical local treatment to all tumour sites, a large number of potential prognosticators was investigated (Table 2). In univariate analysis worse ECOG score (HR 2.8 per point, $p<0.001$ ), the presence of bony $(\mathrm{HR}=7.3, p<0.001)$ and brain metastases (HR 12.5, $p=0.004$ ), higher total tumour volume (HR 1.6 per 100 $\left.\mathrm{cm}^{3}, p=0.005\right)$ as well as higher locoregional tumour volume (HR 1.5 per $100 \mathrm{~cm}^{3}, p=0.029$ ) and higher total metastases volume (HR 2.5, $p=0.031$ ) were significantly associated with worse survival. In contrast the presence of pulmonary metastases $(\mathrm{HR}=0.4, p=0.011)$ was significantly associated with improved survival and higher radiotherapy dose (HR 0.8 per 10 Gy BED $10, p=0.052$ )

Table 2 Univariate Cox's regression analysis of prognostic factors for overall survival $(\mathrm{N}=40)$

\begin{tabular}{|c|c|c|}
\hline \multirow[t]{2}{*}{ Parameter } & \multicolumn{2}{|l|}{ Univariate } \\
\hline & $\mathrm{HR}(95 \% \mathrm{Cl})$ & $p$ value \\
\hline ECOG, per point & $2.8(1.6-4.7)$ & $<0.001$ \\
\hline Bone metastases, yes versus no & $7.3(2.6-20.5)$ & $<0.001$ \\
\hline Brain metastases, yes versus no & $12.5(2.5-69.6)$ & 0.004 \\
\hline Total tumor volume, per $100 \mathrm{~cm}^{3}$ & $1.6(1.2-2.1)$ & 0.005 \\
\hline Pulmonary metastases, yes versus no & $0.4(0.2-0.8)$ & 0.011 \\
\hline Locoregional tumor volume, per $100 \mathrm{~cm}^{3}$ & $1.5(1.0-2.2)$ & 0.029 \\
\hline Total metastases volume, per $100 \mathrm{~cm}^{3}$ & $2.5(1.1-5.7)$ & 0.031 \\
\hline Radiotherapy dose, BED10 per 10 Gy & $0.8(0.7-1.0)$ & 0.052 \\
\hline Number of involved organ systems & $0.4(0.1-1.3)$ & 0.110 \\
\hline $\begin{array}{l}\text { Time interval from OMD diagnosis to first local } \\
\text { treatment, months }\end{array}$ & $0.8(0.6--1.1)$ & 0.170 \\
\hline Number of metastases & $0.8(0.6-1.1)$ & 0.189 \\
\hline Systemic treatment, yes versus no & $1.6(0.8-3.3)$ & 0.229 \\
\hline Cetuximab, yes versus no & $1.7(0.6-4.5)$ & 0.298 \\
\hline Distant nodal metastases, yes versus no & $0.7(0.3-1.6)$ & 0.341 \\
\hline PET Staging & $0.7(0.3-1.6)$ & 0.398 \\
\hline Hepatic metastases, yes versus no & $0.6(0.2-2.0)$ & 0.407 \\
\hline Duration of local treatments for $\mathrm{OMD}$, months & $0.9(0.8-1.1)$ & 0.454 \\
\hline Age, per 10 years & $1.2(0.8-1.7)$ & 0.460 \\
\hline Metachronous versus synchronous OMD & $0.8(0.4-1.6)$ & 0.502 \\
\hline Checkpoint inhibitor treatment, yes versus no & $1.6(0.4-6.6)$ & 0.553 \\
\hline Active locoregional tumor manifestation & $1.2(0.6-2.6)$ & 0.574 \\
\hline $\begin{array}{l}\text { Platin combination chemotherapy, yes versus } \\
\text { no }\end{array}$ & $0.9(0.5-2.0)$ & 0.859 \\
\hline
\end{tabular}

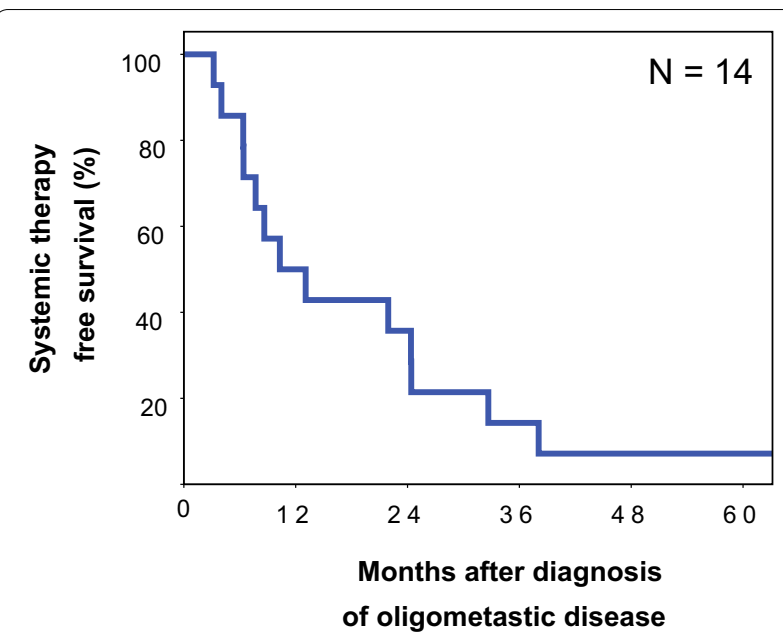

Fig. 3 Systemic therapy-free survival in the subgroup of patients, in which systemic therapy was deferred after local treatment of metastases $(\mathrm{N}=14)$

showed a trend towards significantly improved prognosis. Conversely neither the number of metastases $(p=0.189)$, nor the number of involved organ sites $(p=0.110)$ and age $(p=0.460)$ were significant prognosticators in the present cohort. Similarly, there was no significant difference in survival between patients with synchronous oligometastatic disease and metachronous oligorecurrence $(p=0.502)$ (Table 2).

In multivariate analysis for overall survival, better ECOG status, lower total tumour volume and the absence of brain as well as bony metastases remained significant predictors of improved survival following radical local treatment of all tumour sites (Fig. 4).

\section{Discussion}

Treatment outcomes in metastatic head and neck cancer treated with current systemic treatment options remain unsatisfactory. Platinum-based chemotherapy plus cetuximab achieved a median overall survival of 10.1 months in the well-known study by Vermorken et al. [12]. More recently the addition of pembrolizumab to platinum and 5-FU significantly improved overall survival in the total population of the KEYNOTE-048 trial over cetuximab with platinum and 5-FU (13.0 vs. 10.7 months). In the subset of patients with a PD-L1 combined positive score (CPS) of $\geq 1$ and $\geq 20$ the benefit of pembrolizumab + chemotherapy was more pronounced, achieving a median overall survival of 13.6 and 14.7 months, respectively [13]. Considering that treatments in our study occurred mostly before the introduction of immune checkpoint inhibitors into routine clinical practice, the median overall survival of 23.0 months achieved in the present study with an increased fraction of patients alive 


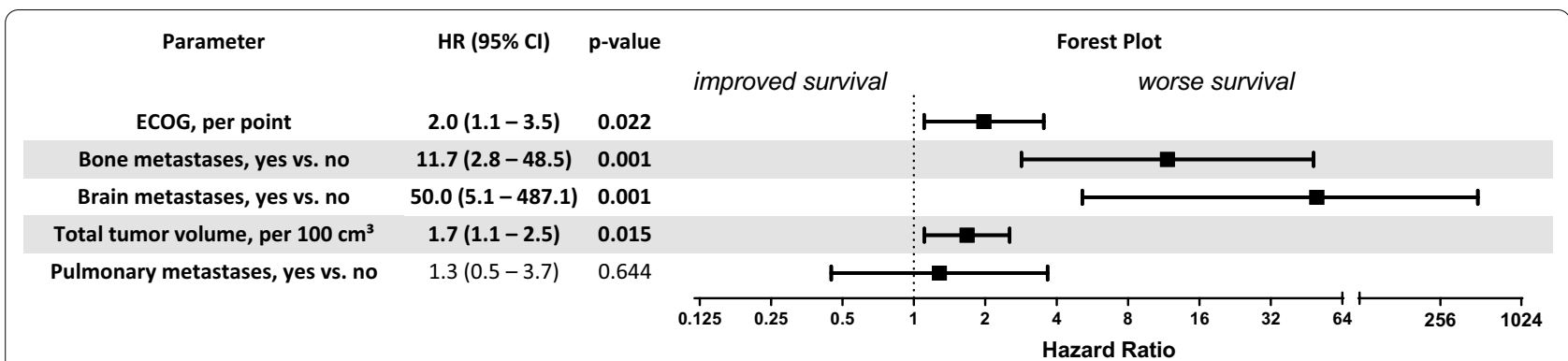

Fig. 4 Multivariate Cox's regression analysis and Forrest plot of prognostic factors for overall survival following radical local treatment of all tumour sites

after 2 years has to be considered as a clear indication that the oligometastatic treatment paradigm holds promise in metastatic head and neck cancer. While selection effects cannot be fully discarded in a retrospective setting, it has to be noted, that over half of patients treated in the Phase III trial by Vermorken et al. suffered from locoregionally recurrent tumour only while all patients in the present cohort had metastatic disease. Similarly, only up to $12 \%$ of patient in the Vermorken trial had a Karnofsky score of less than $80 \%$ while $43 \%$ of patients in the present cohort were characterized by an ECOG score of 2 or worse (i.e., Karnofsky score of < 80\%) [12].

Very few series on oligometastatic head and neck cancer have been reported so far in the literature. Schulz et al. reported on a cohort of 37 patients with metastatic head and neck cancer, in which distant metastases were treated specifically with either surgery or stereotactic body radiotherapy (SBRT) [14]. Observing a median overall survival of 23.97 months, their outcome was remarkably similar to the one achieved in our series. Similarly, Bates et al. reported a median overall survival of 22.8 months in a cohort of patients with oligometastatic head and neck cancer ( $\leq 5$ metastases) treated with SBRT. Therefore, published series on oligometastatic head and neck cancer show a very consistent median overall survival of 23-24 months. Series that are limited exclusively to patients with pulmonary oligometastases form a notable exception to this rule, however. Bonomo et al. for instance achieved a median overall survival of 47 months in a cohort of patients with metastatic head and neck cancer limited to the lungs [15] and Pasalic similarly achieved a median overall survival of around 48 months (value obtained from Kaplan-Meier plot) in a cohort of metastatic head and neck cancer patients with up to 3 lung-only metastases treated with SBRT to all tumour sites [16]. The improvement in overall survival that is consistently observed with local treatment of oligometastatic disease indicates that local treatments may affect systemic disease progression in patients with metastatic cancer. The fact that freedom from new distant metastases ceased to decline after 3 years with $22 \%$ of patients remaining free from new distant metastases at 4- and 5-years post diagnosis of oligometastatic disease in the present series is an interesting observation in this regard. Resembling the finding of decreased distant metastasis formation in NSCLC patients in the Phase II study by Gomez et al., a similar systemic impact of local treatment could also mediate improvements in overall survival in patients with oligometastatic head and neck cancer [5].

An important aim of the present study was to identify prognostic factors for improved survival with radical local treatment and to determine which patients with metastatic head and neck cancer should receive local treatment of all tumour sites. The exploration of prognostic parameters in oligometastatic head and neck cancers treated in radical intent at all tumour sites had only been partially addressed by previous studies. As such, Bates et al. investigated several prognostic factors in a cohort of 27 radically treated patients including number of metastases and involved organ sites but did not find pre-treatment factors that were significantly associated with overall survival [17]. Schulz et al. among others investigated prognostic factors in large cohorts of patient with metastatic head and neck cancers with and without local treatment to all tumour sites [14]. While, importantly all these studies were able to show the prognostic advantage of local treatment and limited metastatic burden, predictors of outcome of patients that actually received radical local treatment was outside the scope of previous series. In the present study, we explored a large number of potential prognosticators first in univariate analysis and included significant parameters subsequently in the final multivariate model. In univariate analysis, we found worse ECOG score and the presence of bone and brain metastases to be negative predictors of overall survival following radical local treatment of all tumour sites in metastatic 
head and neck cancer. Conversely and in line with previous series, the presence of pulmonary metastases was associated with improved survival in univariate analysis. Moreover, we explored the impact of volume-based metrics on overall survival in oligometastatic head and neck cancer. Total tumour volume and other volumerelated parameters have frequently been hypothesized as potential selection criteria for the definition of oligometastatic disease but had not been investigated in oligometastatic head and neck cancer so far $[1,2]$. We used an AI-based autosegmentation approach to segment all tumour sites on baseline imaging for calculation of tumour volumes in every patient. Interestingly, total tumour volume was in fact strongly associated with survival in oligometastatic head and neck cancer. In multivariate analysis, lower total tumour volume, better ECOG status and the absence of brain and bony metastases remained significant predictors for improved survival following radical local treatment of all tumour sites. These results could help in identifying patients with metastatic head and neck cancer who benefit from an oligometastatic treatment paradigm and should be further evaluated in future studies.

A particularly interesting scientific question is whether local treatment could be used to delay initiation of systemic treatment in oligometastatic disease. In our cohort, systemic treatment had been deferred in a subset of $35 \%$ of patients consisting mainly of patients with solitary lung metastasis. In this subgroup, 1-year systemic treatment-free survival was $50 \%$ with 2 - and 3 -year systemic treatment-free survival being $36 \%$ and $14 \%$, respectively. In a retrospective series on lung oligometastases from different primaries, Mazzola et al. showed that lung SBRT achieved a median systemic treatment-free survival of 16 months [18]. In the setting of oligorecurrent prostate cancer, the well-known randomized Phase II STOMP trial by Ost et al. demonstrated a significantly longer androgen deprivation therapy-free survival for metastasis-directed therapy versus observation [7]. Also, in a large multicentre retrospective study by Triggiani et al., the authors demonstrated a promising 1-year systemic treatment-free survival of $72.1 \%$ in oligoprogressive castration-resistant prostate cancer [19]. Collectively, these results indicate a systemic effect of local treatment in oligometastatic disease, and that metastasis-directed therapy is able to substantially delay the initiation of systemic treatment in these patients. However, whether the combination of local treatment with upfront systemic treatment for oligometastatic disease provides additional benefit over delayed administration and in which patients systemic treatment can be safely deferred needs additional prospective and randomized trials to be answered definitely.
While patients in the present series were largely treated before the current era of checkpoint inhibitors, advances in immunotherapies are an important consideration for future studies on oligometastatic head and neck cancer. As synergistic effects for the combination of radiotherapy and checkpoint inhibitor treatment have been described [20-22], the synthesis of local treatment and systemic immunotherapy could be especially beneficial in the oligometastatic setting. Ongoing trails like IMPORTANCE (NCT03386357) and CheckRad-CD8 (NCT03426657) are already investigating optimal combination strategies of radiotherapy and checkpoint inhibitor treatment and results are eagerly awaited.

\section{Limitations}

Being a retrospective study hidden selection effects could have influenced results. The small patient number was a limitation that precluded detailed subgroup analyses and resulted in reduced statistical power.

\section{Conclusions}

Radical local treatment in oligometastatic head and neck cancer showed promising outcomes in this study and clearly warrants further research. Patients with favourable performance status, absence of brain and bone metastases and low total tumour volume were identified as optimal candidates for radical local treatment in oligometastatic head and neck cancer and should be considered for selection in future prospective trials.

\section{Supplementary Information}

The online version contains supplementary material available at https://doi. org/10.1186/s13014-021-01790-w.

Additional file 1: Detailed listing of treatment for de-novo oligometastatic disease and corresponding tumor locations in all patients.

\section{Acknowledgements \\ Not applicable.}

\section{Authors' contributions}

FP, TW, DH, SL, MH, SS and RF conceptualized the manuscript. FP, TW, DH, $\mathrm{MH}$ and IF investigated the findings. FP, TW and IF performed the analysis. FP, $\mathrm{MH}, \mathrm{SS}, \mathrm{BF}, \mathrm{CB}, \mathrm{SL}, \mathrm{KM}, \mathrm{HI}$, and RF provided the resources. FP, TW, MH, SS, IF, BF, $\mathrm{CB}, \mathrm{SL}, \mathrm{KM}$, and RF performed the writing. FP, KM, $\mathrm{HI}$ and RF supervised the findings. All authors contributed to the article and approved the submitted version. All authors read and approved the final manuscript.

\section{Funding}

Open Access funding enabled and organized by Projekt DEAL. FP was supported by a grant from the Interdisciplinary Centre for Clinical Research (IZKF) Erlangen: rotation program for physician scientists (https://www.izkf.med.fau. de/). 


\section{Availability of data and materials}

The raw data supporting the conclusions of this article will be made available by the authors, without undue reservation.

\section{Declarations}

Ethics approval and consent to participate

Ethical review and written informed consent was not required for participation in this retrospective analysis in accordance with the local legislation (BayKrG Art. 27 (4)) and institutional requirements. Written informed consent for treatment was provided by all patients.

\section{Consent for publication}

Not applicable.

\section{Competing interests}

The authors report no conflicts of interest.

\section{Author details}

${ }^{1}$ Department of Radiotherapy, Friedrich-Alexander-Universität ErlangenNürnberg, Universitaetsstraße 27, 91054 Erlangen, Germany. ${ }^{2}$ Department of Otolaryngology, Head and Neck Surgery, Friedrich-Alexander-Universität Erlangen-Nürnberg, Erlangen, Germany.

Received: 27 December 2020 Accepted: 17 March 2021

Published online: 31 March 2021

\section{References}

1. Lievens Y, Guckenberger M, Gomez D, Hoyer M, lyengar P, Kindts I, et al. Defining oligometastatic disease from a radiation oncology perspective: an ESTRO-ASTRO consensus document. Radiother Oncol. 2020;148:157-66.

2. Guckenberger M, Lievens Y, Bouma AB, Collette L, Dekker A, deSouza NM, et al. Characterisation and classification of oligometastatic disease: a European Society for Radiotherapy and Oncology and European Organisation for Research and Treatment of Cancer consensus recommendation. Lancet Oncol. 2020;21(1):e18-28.

3. Clerici E, Comito T, Franzese C, Di Brina L, Tozzi A, Iftode C, et al. Role of stereotactic body radiation therapy in the treatment of liver metastases: clinical results and prognostic factors. Strahlentherapie und Onkologie: Organ der Deutschen Rontgengesellschaft [et al]. 2020;196(4):325-33.

4. Hellman S, Weichselbaum RR. Oligometastases. J Clin Oncol. 1995;13(1):8-10.

5. Gomez DR, Tang C, Zhang J, Blumenschein GR Jr, Hernandez M, Lee Jر J J et al. Local consolidative therapy vs. maintenance therapy or observation for patients with oligometastatic non-small-cell lung cancer: long-term results of a multi-institutional, phase II, randomized study. J Clin Oncol. 2019;37(18):1558-65.

6. lyengar P, Wardak Z, Gerber DE, Tumati V, Ahn C, Hughes RS, et al. Consolidative radiotherapy for limited metastatic non-small-cell lung cancer: a phase 2 randomized clinical trial. JAMA Oncol. 2018;4(1):e173501.

7. Ost P, Reynders D, Decaestecker K, Fonteyne V, Lumen N, De Bruycker A, et al. Surveillance or metastasis-directed therapy for oligometastatic prostate cancer recurrence: a prospective, randomized, multicenter phase II trial. J Clin Oncol. 2018;36(5):446-53.

8. Palma DA, Olson R, Harrow S, Gaede S, Louie AV, Haasbeek C, et al. Stereotactic ablative radiotherapy versus standard of care palliative treatment in patients with oligometastatic cancers (SABR-COMET): a randomised, phase 2, open-label trial. Lancet. 2019;393(10185):2051-8.

9. Fedorov A, Beichel R, Kalpathy-Cramer J, Finet J, Fillion-Robin JC, Pujol $\mathrm{S}$, et al. 3D Slicer as an image computing platform for the quantitative imaging network. Magn Reson Imaging. 2012;30(9):1323-41.

10. van Griethuysen JJM, Fedorov A, Parmar C, Hosny A, Aucoin N, Narayan $\checkmark$, et al. Computational radiomics system to decode the radiographic phenotype. Cancer Res. 2017;77(21):e104-7.

11. Eisenhauer EA, Therasse P, Bogaerts J, Schwartz LH, Sargent D, Ford R, et al. New response evaluation criteria in solid tumours: revised RECIST guideline (version 1.1). Eur J Cancer (Oxf, Engl: 1990). 2009;45(2):228-47.

12. Vermorken JB, Mesia R, Rivera F, Remenar E, Kawecki A, Rottey S, et al. Platinum-based chemotherapy plus cetuximab in head and neck cancer. N Engl J Med. 2008;359(11):1116-27.

13. Burtness $B$, Harrington $K J$, Greil R, Soulières $D$, Tahara M, de Castro G Jr, et al. Pembrolizumab alone or with chemotherapy versus cetuximab with chemotherapy for recurrent or metastatic squamous cell carcinoma of the head and neck (KEYNOTE-048): a randomised, open-label, phase 3 study. Lancet. 2019;394(10212):1915-28.

14. Schulz D, Wirth M, Piontek G, Knopf A, Straube C, Pigorsch S, et al. Improved overall survival in head and neck cancer patients after specific therapy of distant metastases. Eur Arch Oto-rhino-laryngol. 2018;275(5):1239-47.

15. Bonomo P, Greto D, Desideri I, Loi M, Di Cataldo V, Orlandi E, et al. Clinical outcome of stereotactic body radiotherapy for lung-only oligometastatic head and neck squamous cell carcinoma: is the deferral of systemic therapy a potential goal? Oral Oncol. 2019;93:1-7.

16. Pasalic D, Betancourt-Cuellar SL, Taku N, Ludmir EB, Lu Y, Allen PK, et al. Outcomes and toxicities following stereotactic ablative radiotherapy for pulmonary metastases in patients with primary head and neck cancer. Head Neck. 2020;42(8):1939-53.

17. Bates JE, De Leo AN, Morris CG, Amdur RJ, Dagan R. Oligometastatic squamous cell carcinoma of the head and neck treated with stereotactic body ablative radiotherapy: single-institution outcomes. Head Neck. 2019:41(7):2309-14.

18. Mazzola R, Fersino S, Ferrera G, Targher G, Figlia V, Triggiani L, et al. Stereotactic body radiotherapy for lung oligometastases impacts on systemic treatment-free survival: a cohort study. Med Oncol. 2018;35(9):121.

19. Triggiani L, Mazzola R, Magrini SM, Ingrosso G, Borghetti P, Trippa F, et al. Metastasis-directed stereotactic radiotherapy for oligoprogressive castration-resistant prostate cancer: a multicenter study. World J Urol. 2019:37(12):2631-7.

20. Weichselbaum RR, Liang H, Deng L, Fu YX. Radiotherapy and immunotherapy: a beneficial liaison? Nat Rev Clin Oncol. 2017;14(6):365-79.

21. Rückert M, Deloch L, Fietkau R, Frey B, Hecht M, Gaipl US. Immune modulatory effects of radiotherapy as basis for well-reasoned radioimmunotherapies. Strahlentherapie und Onkologie: Organ der Deutschen Rontgengesellschaft [et al]. 2018;194(6):509-19.

22. Frey B, Mika J, Jelonek K, Cruz-Garcia L, Roelants C, Testard I, et al. Systemic modulation of stress and immune parameters in patients treated for prostate adenocarcinoma by intensity-modulated radiation therapy or stereotactic ablative body radiotherapy. Strahlentherapie und Onkologie. 2020;epub before print.

\section{Publisher's Note}

Springer Nature remains neutral with regard to jurisdictional claims in published maps and institutional affiliations. 